\title{
O Agente Pedagógico Animado MInA
}

\author{
Vandor Roberto Vilardi Rissoli ${ }^{1}$, Giovanni Almeida Santos ${ }^{2}$ \\ ${ }^{1}$ Universidade Católica de Brasília (UCB) \\ QS07 Lote 01 EPCT - Fone: 6133569306 - 72002-900 Taguatinga - DF \\ ${ }^{2}$ Universidade de Brasília (UnB) \\ Área Especial de Indústria Projeção A - Fone: 6131078901 - 72444-240 Gama - DF \\ Apoio: Conselho Nacional de Desenvolvimento Científico e Tecnológico (CNPq) \\ vandoraucb.com, giovannixaunb.br
}

\begin{abstract}
This paper presents specifications of an artificial intelligent agent to be integrated into an Intelligent Tutoring Systems, applied as a methodology to support teaching and learning of graduate disciplines. This integration involves technological resources from the Artificial Intelligence aiming to model the cognitive status of each learner and use resources that are able to work emotional and affective aspects of motivation in an educational process, making it more pleasant and efficient.
\end{abstract}

Resumo. Este artigo apresenta especificações de um agente artificial inteligente a ser integrado a um Sistema Tutor Inteligente, aplicado como metodologia de apoio ao ensino-aprendizagem em disciplinas de cursos de graduação. Esta integração tecnológica envolve recursos provenientes da Inteligência Artificial almejando modelar a situação cognitiva de cada aprendiz e empregar recursos emotivos que sejam capazes de trabalhar aspectos afetivos e de motivação em um processo educacional, procurando torná-lo mais eficiente.

\section{Introdução}

O uso do computador e da Internet é cada vez mais comum e até essencial no dia a dia das pessoas na sociedade atual. $O$ acesso às tecnologias da informação tem se intensificado não apenas com propósitos técnicos, no âmbito do mercado de trabalho, mas de fato no cotidiano das pessoas como ferramentas que proporcionam melhores condições de vida, simplificando a rotina diária, ampliando a comunicação, maximizando o tempo e suas potencialidades.

Esse comportamento mostra que as tecnologias também têm sido utilizadas como ferramentas de socialização e não apenas como recurso simples de acesso e busca de dados e informações. Assim, os ambientes computacionais estão sendo projetados para facilitar a interação com seus usuários e auxiliá-los na comunicação com outras pessoas. É possível ainda observar a tendência contínua desses projetos em "humanizar" seus recursos interativos, almejando uma evolução no sentido do afastamento das perspectivas convencionais de programação centradas nos dispositivos computacionais, para uma outra perspectiva mais sintonizada as realidades humanas, sendo baseada em conceitos e metáforas próximas da visão humana do mundo [Wooldridge 2002]. 
Um exemplo dessa realidade é a projeção de interfaces limpas, com comandos e instruções de caráter emocionalmente mais agradáveis nas redes sociais. Geralmente, esses ambientes virtuais são dotados de ferramentas de software que possibilitam aos seus usuários expressarem seu humor, suas ideias e emoções. A utilização de personagens virtuais nessas interfaces tem se tornado comum, pois facilita e agiliza a interação expressivamente segura entre seus usuários. Esta tendência, da humanização cada vez maior dos sistemas computacionais que envolvem aspectos emotivos, torna a experiência no uso desses recursos tecnológicos mais interativas e amigáveis, elevando a motivação de seus usuários.

Essas características interativas, de se manter o usuário interessado e motivado a prosseguir em sua atividade mediada por tecnologia, é um desafio para diversas áreas de conhecimento, em especial à Educação. No processo ensino-aprendizagem, a motivação precisa ser mantida em alto nível pelo professor e compartilhada por seus aprendizes. Dentro dessa perspectiva, a preocupação do ensino tem sido a de criar condições para que o aluno deseje aprender, pois, através da motivação, o indivíduo sente vontade de entender os conteúdos envolvidos e emprega o esforço necessário para tal assimilação durante o período de tempo planejado para se atingir o objetivo proposto [Bock 1999].

Consciente desta realidade, este artigo apresenta as especificações usadas na elaboração de um agente artificial inteligente a ser integrado ao Sistema Tutor Inteligente conhecido como SAE (Sistema de Apoio Educacional), aplicado como recurso de apoio à metodologia educacional das disciplinas iniciais da área de Programação Computacional em um curso de Ciência da Computação. Este STI emprega técnicas de Inteligência Artificial (IA) a fim de identificar as dificuldades de aprendizagem de cada indivíduo, fornecendo-lhes orientação sob medida para tal superação. A integração com esse novo agente almeja efetivar uma interação mais agradável com seus usuários, envolvendo aspectos emotivos no trabalho de motivação e orientação pedagógica de alunos.

Este artigo está organizado em 6 seções. Na seção 2 são sintetizados alguns conceitos fundamentais sobre agentes inteligentes e pedagógicos. A seção 3 aborda as principais características do agente MInA, enquanto que na seção 4 são mostrados alguns de seus resultados. A seção 5 apresenta as conclusões deste trabalho, sendo relacionadas ao final suas principais referências bibliográficas.

\section{Agentes Inteligentes}

O conceito de agente inteligente surge a partir da modelagem, por parte dos avanços na área da IA, de uma entidade que materialize, em meio virtual, a concepção de um ser inteligente. Compreende-se como um agente inteligente um software, ou um módulo de um software, que seja capaz de organizar, selecionar, produzir informações e tomar decisões a partir de alguma fonte de dados. Assim, um agente é um subsistema criado com a finalidade de reter e analisar dados, sendo capaz de responder adequadamente a uma possível situação analisada. Por meio da tecnologia de agentes é possível uma reflexão a respeito da natureza da interação entre o ser humano e o computador, onde este último assume o papel de parceiro de seu usuário humano, cooperando para o alcance de seus objetivos [Menezes 1998].

Apesar do uso frequente do termo "agente" na IA, não existe um consenso estrito a respeito da sua definição. São encontradas diversas classificações, das mais 
simples às mais complexas. No entanto, existem algumas características esperadas em um módulo computacional para que este seja reconhecido como agente inteligente. Entre estas podem ser apontadas as mais básicas descritas por Jennings e Wooldridge (1998): i) Autonomia: atuação do agente sem intervenção direta humana ou de outros agentes, possuindo um nível de controle sobre o seu estado interno e suas ações; ii) Próatividade: capacidade de ação não apenas em resposta aos estímulos do ambiente que o envolve, mas também buscando atingir seus objetivos; iii) Reatividade: capacidade de detecção de mudanças em seu ambiente e de reação coerente; iv) Sociabilidade: possibilidade de interação com outros agentes, artificiais ou humanos, para atingir objetivos.

Com o passar do tempo, outros tipos e propostas de classificação surgiram, levando em consideração o propósito e a capacidade funcional desses agentes, como aqueles que desenvolvem atividades conversacionais, sociais, pedagógicas, entre outras. Quando estão inseridos em um ambiente educacional, com objetivos voltados ao apoio do ensino-aprendizagem, são classificados como agentes pedagógicos, tendo atuação no acompanhamento das atividades discentes, monitoramento no desenvolvimento de tarefas, identificação de dificuldades dos aprendizes, fornecimento de esclarecimentos, além do auxílio na resolução de problemas. Tornam-se, assim, tutores artificiais ou assistentes virtuais que fornecem suporte aos seus usuários-estudantes.

\subsection{Agentes Pedagógicos}

O uso de agentes pedagógicos em softwares educacionais traz elementos relacionados à personificação, visibilidade e personalidade, configurando o "efeito persona" proposto em Lester (1997). Esse efeito contribui para o relacionamento pessoal e emotivo do aluno com o agente, promovendo o maior interesse e motivação à interação do aprendiz com seu ambiente virtual (ambiente de software em que o agente atua) [Gulz 2006].

Os agentes pedagógicos podem se apresentar ao usuário de forma gráfica, como um personagem animado, usando recursos multimídia para prover uma interação mais visual. As entidades que assumem essa característica são conhecidas como agentes pedagógicos animados. Nos ambientes virtuais de ensino estes agentes têm-se tornado uma tendência, pois através de uma interface animada é possível direcionar a atenção do aprendiz por meio de gestos e movimentos, conduzindo-o aos aspectos mais importantes das tarefas propostas e à compreensão das possíveis orientações [Johnson 2000] .

Dessa forma, torna-se possível a ampliação dos meios de comunicação do ambiente virtual de aprendizagem com seus estudantes, sendo empregados recursos tecnológicos que reforçam e esclarecem o aprendiz de maneira personalizada e acolhedora, motivando-o a uma experiência mais agradável e interativa.

Nos avanços da pesquisa e desenvolvimento de agentes inteligentes, as características antropomórficas têm sido cada vez mais inseridas nesses agentes, tornando-os mais sensíveis e expressivos as linguagens humanas de interação, ressaltando as possibilidades de comunicação por meio de metáforas, situações peculiares ao cotidiano e a cultura, movimentação corporal, além dos aspectos afetivos e emocionais.

Morgado (2006) defende o uso de capacidades cognitivas em agentes inteligentes, com modelos que sirvam de suporte à implementação de mecanismos de regulação e adaptação dos processos de raciocínio e tomada de decisão. Assim, a interação entre agente e usuário humano assumiria um caráter mais pessoal e intuitivo, 
numa abstração superior a uma simples interação com um programa de computador. Dentre as atividades previstas para este tipo de interação estão as expressões faciais em personagens virtuais, que poderiam transmitir estados emocionais, demonstrando afeto e amizade, além da inclusão de uma personalidade que se assemelhe às características humanas e possa assimilar convenções sociais, postura no trato com crianças e idosos, inclusive, demonstrando sentimento com cada um deles [Bercht 2001].

Piaget (2005) destaca o quanto o aspecto afetivo é fator energético para o desenvolvimento intelectual utilizando a metáfora da gasolina e do automóvel - onde essa "energia" pode influenciar o "funcionamento" da aprendizagem.

A afetividade compreende o domínio das emoções, de seus sentimentos e experiências que possam promover o contato com sensações a um indivíduo em determinado instante, sendo este conjunto de características sentimentais denominado estado afetivo [Bercht 2001]. Em um processo educacional, a identificação desses estados nos aprendizes pode permitir que ações sejam tomadas a fim de auxiliá-los no andamento dos seus estudos, promovendo um ensino-aprendizagem mais agradável e sintonizado às características emotivas de cada estudante [Longhi 2010].

Nesse sentido, diversos trabalhos ([Frozza 2009], [Bocca 2003], ...) têm sido realizados com o uso de agentes pedagógicos animados que exploram características humanas nas entidades computacionais. O ambiente MACES (Multiagent Architecture for an Collaborative Educational System) exemplifica esta situação, pois possui o agente pedagógico animado PAT (Pedagogical and Affective Tutor) mediando sua operação [Bocca 2003]. Esse agente teve sua aparência definida (diagramada) após pesquisas e entrevistas realizadas com pedagogos, psicólogos, psicopedagogos e designers.

Dessa forma, um agente inteligente, adequadamente elaborado, consiste em recurso tecnológico que vai além de um software, pois adquire personalidade e pode fornecer confiança e motivação no uso do ambiente computacional em que esteja inserido. Sua aplicação em processos educacionais pode colaborar com o ensino e tornar a aprendizagem mais agradável e intuitiva aos seus estudantes.

\section{O Agente MInA}

Um recurso fundamental no desenvolvimento de agentes pedagógicos animados é o projeto de interação do agente com os seres humanos, priorizando a transmissão de aspectos afetivos e emocionais. Esses aspectos são trabalhados por uma área da IA conhecida como Computação Afetiva, que estuda as emoções e suas possíveis aplicações em sistemas computacionais [Picard 1997].

Normalmente, o sucesso no ensino-aprendizagem está relacionado, diretamente, com estados afetivos dos alunos, podendo causar interferência na motivação e na aptidão em se aprender algo. Aspectos visuais e afetivos são relevantes ao projeto de um agente, sendo o Mangá, técnica de quadrinhos japoneses, destacado como poderoso recurso de transmissão de ideias e sentimentos, com uma forte abordagem visual [Luyten 2000].

Essa arte de elaboração de quadrinhos tem seu estilo originário dos rolos de pintura desde o século XII d.C, com ilustrações e textos que narravam uma história 
enquanto eram desenrolados. É frequente nessas obras a ocorrência de textos explicativos após extensas cenas de pintura, o que evidencia a priorização da imagem sobre o texto, assegurando sozinha a narração. Esta característica perpetua-se até os dias atuais como uma das mais importantes do Mangá.

O sucesso das histórias narradas por intermédio de ilustrações vem do interesse perene do figurativo na cultura oriental. A própria escrita japonesa, com os ideogramas, tem a tradição de abstrair traços de figuras reais, ou seja, signos que exprimem a ideia das palavras. A escrita alfabética, predominante no mundo ocidental, não transmite nenhum sentido de imediato. É preciso decodificar as palavras em conceitos para chegar ao sentido desejado e compreendê-la. Porém, a estética do Mangá atual surgiu no século XX, com a influência dos desenhos e da cultura proveniente dos Estados Unidos e da Europa, que foram impostos ao Japão após a Segunda Guerra Mundial. Com as obras do artista Osamu Tezuka, pai do Mangá moderno, essa técnica adquiriu seu traçado moderno, onde a expressividade dos personagens é ampliada pelo desenho exagerado dos olhos, boca, sobrancelhas e nariz. Atualmente, o Mangá é um fenômeno além das fronteiras japonesas, conquistando um público diversificado através da sua variedade de temas, com histórias para os mais variados gêneros, faixas etárias e gostos.

Todas estas características influenciaram na modelagem deste novo agente, elaborado em Mangá, que passou a atuar no ambiente do SAE e é mostrado na Figura 1.

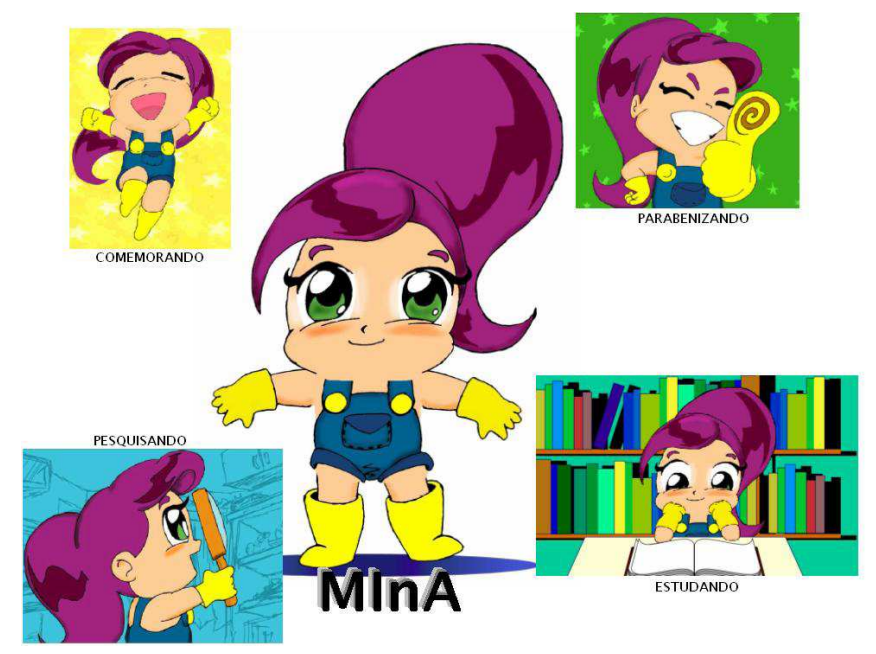

Figura 1. Modelo gráfico do agente MInA e a indicação de algumas animações.

As principais diretrizes desse projeto foram: $i$ ) projetar uma entidade feminina que confere maior receptividade aos usuários masculinos e femininos, principalmente pelos aspectos afetivos relacionados a esse gênero humano [Carter 2003]; ii) dotá-lo de expressões faciais e corporais variadas, compartilhando emoções como alegria, tristeza, susto e outras; iii) trajá-lo com roupas e adereços típicos de um ambiente de labor significativo ao contexto de trabalho e estudo de seus usuários para colaborar na percepção da necessidade de ação, esforço e pró-atividade; $i v$ ) mostrar uma fisionomia limpa, jovial e alegre; v) usar cores quentes em sua composição, que priorizam motivação e disposição; vi) interagir com usuário por meio de gestos, balões e sons, assegurando que a experiência com o ambiente jamais se torne monótona; vii) usar de frases e metáforas que informe ao aluno a sua situação de aprendizagem, de forma realista e motivadora, posicionando o agente como assistente e companheiro nos desafios educacionais. 
Para este agente, apresentado ao usuário como um personagem animado, foi adotado o nome MInA, termo de fácil assimilação, correspondente ao acrônimo de "Módulo de Interface Animado", que também representa as características marcantes da personalidade deste agente: pequena, doce e feminina, como ilustrado na Figura 1.

A MInA se integra ao SAE como mais um módulo que compõe a sua arquitetura, promovendo a participação de vários agentes humanos como colaboradores do ensino-aprendizagem, assistidos pelos recursos interativos deste STI. Suas análises e inferências acontecem sobre os dados coletados durante a interação de seus usuários com suas interfaces destinadas ao suporte de cada perfil participante do processo educacional. Essa situação de assistência também passa a acontecer com a MInA, por meio do fornecimento de dados e relatórios de suporte as interações realizadas por ela e seus estudantes junto as bases de dados e conhecimentos do SAE.

Baseado na Teoria da Aprendizagem Significativa, proposta por Ausubel (1980), este STI acompanha o esforço, o desempenho e a participação de cada estudante, por meio de análises quantitativas e qualitativas que empregam a Lógica Fuzzy no tratamento das incertezas e imprecisões inerentes às informações [Rissoli 2011].

A inferência dos estados afetivos dos alunos é baseada na observação e análise do comportamento interativo de cada aprendiz com os recursos virtuais oferecidos pelo $\mathrm{SAE}$, além do contato com os demais agentes humanos que participam desse processo e são usuários-colaboradores desse STI (outros alunos, monitores e professores).

O uso da Lógica Fuzzy se sobressai à Lógica Convencional na realização desta inferência, principalmente na representação do raciocínio humano, onde sua capacidade de expressão se mostra mais coerente ao tratamento necessário destas imprecisões, possibilitando a sua conversão em informações objetivas e melhores definidas por meio do rigor matemático. Isso eleva o potencial de representação da realidade apurada pelo SAE, sendo esta efetuada em linguagem natural, conforme indicado na Figura 2.
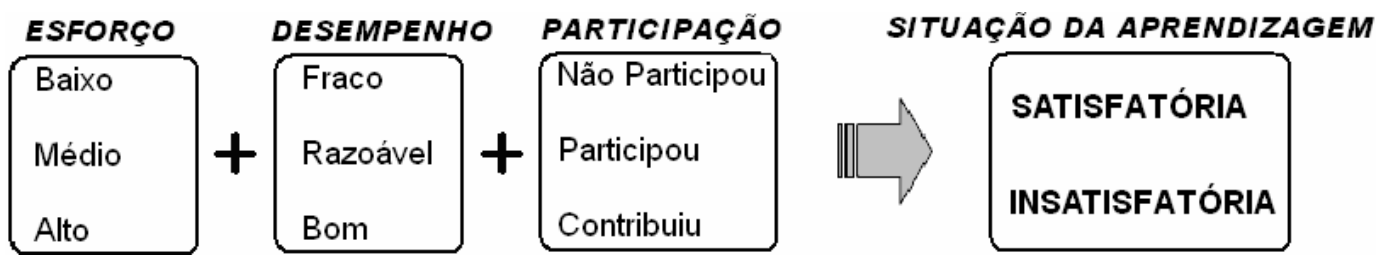

Figura 2. Representação linguística do SAE pela Lógica Fuzzy.

Nesse STI, a função de pertinência, responsável por fornecer um grau de confiança sobre a aprendizagem de cada conceito pertencente a um conteúdo letivo, é elaborada por docentes e especialistas nesse conteúdo. A esta função são atribuídas as características mínimas dos conceitos a serem avaliados por meio de atividades interativas que serão realizadas pelo aluno junto ao SAE durante seu processo de ensino-aprendizagem.

Uma vez definida a função, esta passa a atuar na identificação do estado cognitivo de cada estudante em relação à aprendizagem almejada sobre determinado conteúdo. Para os resultados obtidos em cada conceito relevante de um conteúdo é calculado o seu grau de pertinência (confiança), que indicará, por meio da inferência deste STI, qual é a situação da aprendizagem do estudante, com base no acompanhamento de seu comportamento frente as suas atividades interativas e ao seu possível estado afetivo. 
Diante destas análises, o SAE consegue fornecer orientações pedagógicas adequadas ao estado cognitivo de cada aprendiz em determinado conteúdo, propondo um caminho apropriado de estudo e reforço coerente a sua situação de aprendizagem. Paralelamente, o docente também atua neste direcionamento personalizado do ensinoaprendizagem, munido do acompanhamento dos aprendizes obtido através deste STI. Porém, é imprescindível o comprometimento e o empenho do estudante em seu processo educacional, fazendo sua parte na realização das atividades de estudo e assimilação dos conteúdos orientados por seu docente e este STI preocupado com os aspectos emotivos.

Um reforço no fornecimento das orientações provenientes do SAE é efetivado pelo agente MInA, que almeja realizar uma interação mais emotiva e significativa à compreensão de seus aprendizes sobre a sua atual situação de aprendizagem. Como um colega de turma, esse agente ainda aconselha seus aprendizes a um caminho de estudo que possa ser mais motivador e coerente com a superação de possíveis dificuldades de aprendizagem, conforme representa a Figura 3.

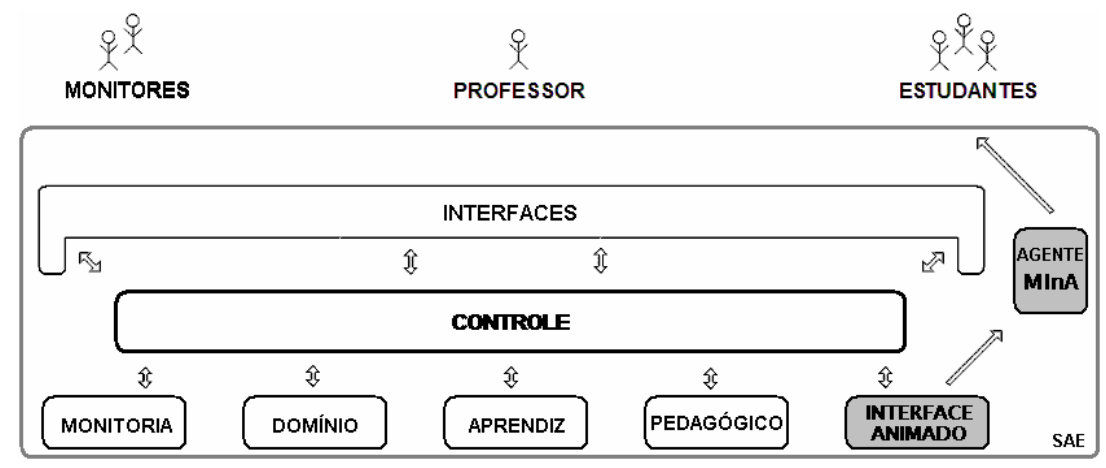

Figura 3. Representação da arquitetura SAE com o agente MInA integrado.

As orientações fornecidas pelo agente são apresentadas por meio de animações com cenas significativas e associadas às situações emotivas que trabalham a constatação da atual situação de aprendizagem envolvendo seus estudantes. Algumas mídias diferentes são aliadas aos textos que fornecem conselhos adequados aos alunos, sendo estes condizentes com as análises mais realistas efetuadas pela Lógica Fuzzy sobre o estado motivacional e cognitivo de cada estudante, diferentemente das apurações mais convencionais que trabalham o acionamento mais algorítmico do agente PAT.

A tecnologia Java para web foi usada na implementação do módulo lógico deste agente, bem como na sua interface de comunicação com o SAE. Sua diagramação gráfica e as animações utilizaram um software específico (Adobe Flash), comumente usado em animações digitais, inclusive em páginas virtuais na Internet. A popularidade desse software garante a acessibilidade de aplicações desenvolvidas nesse STI, onde cerca de $98 \%$ dos computadores conectados à Internet já possuem o seu "executor" (runtime) instalado (Flash Player) [Milward Brown 2009].

Dessa forma, é fornecido ao aprendiz um agente pedagógico animado e emocionalmente sensível, que pode guiá-lo em suas atividades de aprendizagem conforme análises realizadas sobre o seu próprio perfil, que é acompanhado e armazenado pelo SAE. Essa implementação envolveu aspectos emotivos na interação com o estudante, objetivando, principalmente, melhorar a eficiência do processo de 
compreensão do aprendiz sobre qual é a sua situação de aprendizagem detectada por este STI, possibilitando ao aprendiz uma análise reflexiva sobre seus sucessos e dificuldades no contexto do estudo deste conteúdo, bem como nas ações que lhe pareçam mais adequadas ao alcance de seus objetivos.

Associados às possíveis combinações linguísticas detectadas pelo SAE, e representadas na Figura 2, estão vinculados os estados afetivos que serão trabalhados pelo agente MInA, tendo esses estados duas formas principais de influência: a positiva, envolvendo a alegria, felicidade, comemoração e a ação no estudo; ou a negativa com a tristeza e o desalento. Independente da situação e forma de influência detectada pelo SAE, o agente MInA se comunicará com o aprendiz motivando-o ao esforço responsável e com a expectativa relacionada diretamente a sua aprendizagem, a fim de elevar sua motivação a cada nova dificuldade que possa surgir durante sua jornada de estudo.

\section{Resultados}

A análise dos resultados obtidos com a aplicação do SAE sobre duas turmas de uma mesma disciplina (Laboratório 1) do segundo semestre do curso de graduação matutino em Ciência da Computação está sintetizada na Tabela 1. Essa disciplina é presencial, com carga horária de 90 horas em laboratório, sendo a primeira a lecionar uma linguagem de programação (Linguagem $\mathrm{C}$ ). Os seus índices de aprovação em três semestres letivos $(\mathbf{A}=\mathbf{2 0 0 6 / 2}$ último semestre que esta disciplina foi lecionada sem o uso do SAE - conjunto de controle; $\mathbf{B}=2007 / 1$ primeiro semestre usando o SAE; C = 2012/2 semestre mais recente analisado com o uso do SAE) estão na Tabela 1.

Tabela 1. Síntese dos dados comparativos entre três semestres letivos.

Disciplina: LABORATÓRIO 1

\begin{tabular}{|c|c|c|c|c|c|c|c|}
\hline $\begin{array}{l}\text { Docente } \\
\text { / Turma }\end{array}$ & $\begin{array}{c}\text { Semestre A } \\
(\text { sem SAE })\end{array}$ & $\begin{array}{c}\text { Semestre B } \\
(\mathrm{com} S A E)\end{array}$ & $\begin{array}{c}\text { Semestre C } \\
(\mathrm{com} \mathrm{SAE})\end{array}$ & $\begin{array}{l}\text { Difere } \\
A \text { e B }\end{array}$ & $\begin{array}{l}\text { rça Sem } \\
\text { A e C }\end{array}$ & $\begin{array}{l}\text { stral } \\
\text { B e C }\end{array}$ & $\begin{array}{c}\text { Taxa } \\
\text { Evasão }\end{array}$ \\
\hline $\mathrm{D}_{1} / \mathrm{T}_{1}$ & $46,4 \%$ & $65,0 \%$ & $72,2 \%$ & $18,6 \%$ & $25,7 \%$ & $7,2 \%$ & $27,8 \%$ \\
\hline$D_{2} / T_{2}$ & $44,8 \%$ & $54,5 \%$ & $55,6 \%$ & $9,7 \%$ & $10,8 \%$ & $1,1 \%$ & $29,6 \%$ \\
\hline
\end{tabular}

A observação da Tabela 1 indica uma melhora média superior a 18,2\% na aprovação das turmas ( $\mathrm{T}_{1}$ e $\mathrm{T}_{2}$ ) envolvidas neste experimento (estudo de caso). Essa melhora é obtida por meio da comparação do semestre $\mathbf{A}$, que não usou o SAE, em relação ao semestre $\mathbf{C}$, último semestre que o empregou como recurso tecnológico de apoio à aprendizagem e envolveu um contingente de 45 alunos, 2 docentes e 5 monitores.

$\mathrm{O}$ docente $D_{1}$ integrou a sua postura didático-pedagógica os recursos de orientação do SAE e trabalhou com a MInA durante todo período letivo em sua turma, enquanto o docente $D_{2}$ abdicou desse recurso e não utilizou esse agente no SAE. É importante ressaltar a preocupação que envolve a postura pedagógica dos docentes na Tabela 1, que só compara turmas entre semestres diferentes com o mesmo professor.

Em uma pesquisa, realizada junto aos estudantes desta disciplina, no final do semestre letivo de 2012/2, foi possível confirmar que a turma do docente $D_{1}$, que adota a MInA como recurso de apoio educacional, teve maior aprovação e elevada interação com o SAE, onde mais de $92,3 \%$ dos estudantes desta turma, que participaram desta 
pesquisa (72,2\% da turma), solicitaram orientação pedagógica ao STI (Figura 4a). 92\% destes estudantes asseveram que seguiram as orientações (Figura 4b) e 100\% declaram que conhecem a MInA (Figura 4c).

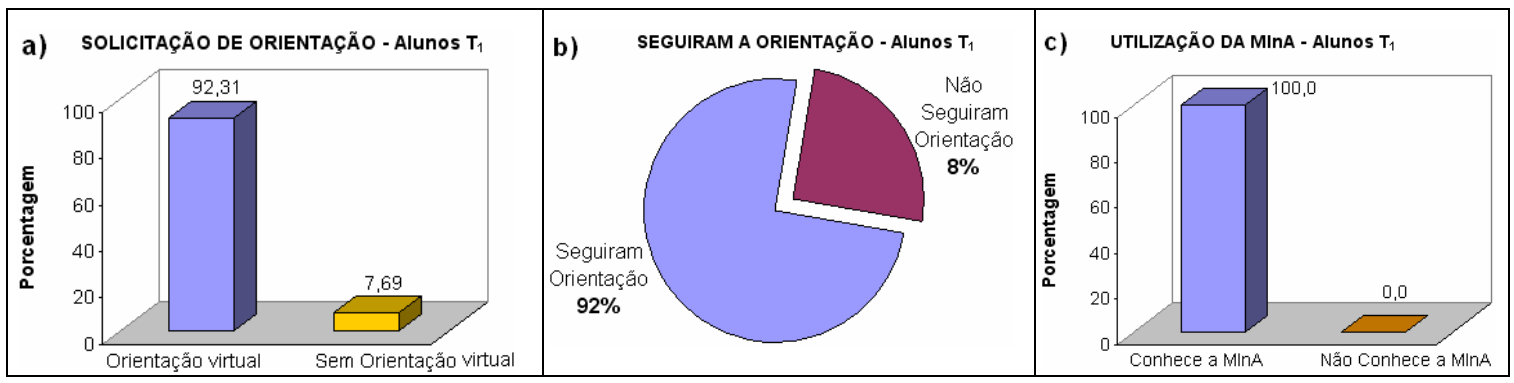

Figura 4. Resultados da pesquisa aplicada aos alunos de Laboratório 1, sendo a) Solicitar Orientação; b) Seguir Orientação; c) Utilizar a MInA.

Ainda na opinião destes estudantes da turma $T_{1}, 84,6 \%$ afirmaram que a MInA os ajudou e motivou durante o ensino-aprendizagem dessa disciplina (Figura 5a). Nos apontamentos dos aprendizes da outra turma $\left(T_{2}\right)$, que teve participação de quase $41 \%$ dos alunos, $100 \%$ dos estudantes relataram que este tipo de recurso (agente pedagógico animado) seria interessante à aprendizagem na disciplina (Figura 5b), onde 54,5\% disseram conhecer algo sobre a MInA (Figura 5c), mas seu professor não a utiliza.
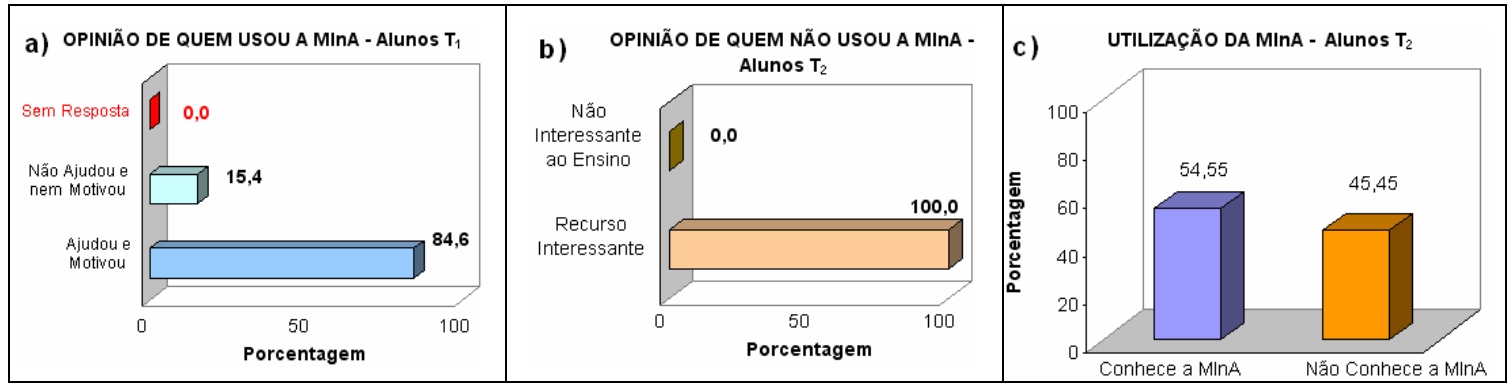

Figura 5. Pesquisa com opinião dos alunos sobre a MInA, sendo a) Agente ajudou e motivou; b) Recurso interessante ao ensino; c) Sabem algo da MInA.

\section{Conclusões}

A principal contribuição deste trabalho consiste na elaboração de um agente pedagógico animado que envolve aspectos afetivos no trato com seus alunos e demonstra o potencial da Lógica Fuzzy neste tipo de inferência. Dentre as expectativas relacionadas ao uso do SAE, com o agente MInA, se destaca o apoio individualizado para um número ilimitado de aprendizes que podem interagir com este agente a qualquer momento e no ritmo que lhes seja mais adequado. Essa realidade propicia um ensino-aprendizagem mais autônomo e personalizado às necessidades de seus aprendizes, acolhendo-os com mais naturalidade e envolvimento emotivo durante as interações com este STI.

Essas análises comparativas indicam perspectivas promissoras ao projeto $\mathrm{SAE}$, que poderá envolver outros agentes inteligentes na interação específica com o docente, além da possibilidade de novas inferências a serem estabelecidas pelo SAE, almejando melhorar a modelagem cognitiva e afetiva de seus estudantes e a eficiência do ensinoaprendizagem nesta área de conhecimento (Programação), além de tentar reduzir suas taxas de evasão que se mantêm elevadas, conforme podem ser observadas na Tabela 1. 


\section{Referências}

Ausubel, D. P.; Novak, J. D.; Hanesian, H. (1980) Psicologia Educacional. Rio de Janeiro: Editora Interamericana.

Bercht, M. (2001) Em Direção a Agentes Pedagógicos com Dimensões Afetivas. Tese (Informática) - Universidade Federal do Rio Grande do Sul, Porto Alegre.

Bocca, E.; Jaques, P.; Vicari, R. (2003) Modelagem e Implementação da Interface para Apresentação de Comportamentos Animados e Emotivos de um Agente Pedagógico Animado. Porto Alegre, Novas Tecnologias na Educação, CINTED-UFRGS, v.1 n.2.

Bock, A. M. B. (org) (1999) Psicologias: uma introdução ao estudo de Psicologia. 13 ed. São Paulo: Saraiva.

Carter, R. (2003) O livro de ouro da mente. Rio de Janeiro: Ediouro.

Frozza, R.; Silva, A. K.; Lux, B.; Cruz, M. E. J. K.; Borin, M. (2009) Dóris 3D: Agente Pedagógico baseado em Emoções. In: SBIE. Florianópolis: SBC.

Gulz, A.; Haake, M. (2006) Design of animated pedagogical agents: A look at their look. In: International Journal of Human-Computer Studies, 64, pp. 322-339.

Jennings, N.; Wolldridge, M. (1998). Applications of Intelligent Agents. In Jennings, N, Wooldridge, M. (eds.), Agent Technology - Foundations, Applications, and Markets. Springer-Verlag.

Johnson, W. L.; Rickel, J. W.; Lester, J. (2000) Animated Pedagogical Agents: Face-toFace Interaction in Interactive Learning Environments. In: International Journal of Artificial Intelligence in Education, 11, pp. 47-78.

Lester, J.; Sharolyn, C.; Kahler, S.; Barlow, T.; Stone, B.; Bhoga, R. (1997) The Persona Effect: Affective Impact of Animated Pedagogical Agents. In: ACM Conference on Human Factors in Computing Systems, pp. 359-366.

Longhi, M.T.; Simonato, G.; Behar, P. A.; Bercht, M. (2010). Um framework para tratamento do léxico afetivo a partir de textos disponibilizados em um ambiente virtual de aprendizagem. CINTED/UFRGS. Porto Alegre, RS.

Luyten, S. B. (2000) Mangá: o poder dos quadrinhos japoneses. São Paulo: Hedra.

Menezes, R.; Fuks, H.; Garcia, A. C. (1998) Utilizando Agentes no Suporte à Avaliação Informal no Ambiente de Instrução Baseada na Web-Aulanet. In: SBIE. Fortaleza: SBC.

Milward Brown Institute (2009) Adobe Survey. Pesquisa realizada em Junho de 2009. Disponível em: <http://www.adobe.com/products/player_census/flashplayer>. Acesso: jan. 2013.

Morgado, L. F. G. (2006) Integração de Emoção e Raciocínio em Agentes Inteligentes. Tese (Informática) - Universidade de Lisboa, Portugal.

Piaget, J. (2005). Inteligencia y afectividad. Buenos Aires: Aique.

Picard, R. (1997) Affective Computing. Cambridge: MIT Press.

Rissoli, V. R. V.; Santos, G. A. (2011) Um Assistente Inteligente Fuzzy no acompanhamento da Aprendizagem Significativa. In: ENIA, SBIE. Natal: SBC.

Wooldridge, M. (2002) An Introduction to MultiAgent Systems. Jon Wiley \& Sons. 